\title{
Diversidad del género Drosophila (Diptera, Drosophilidae) en la quebrada de Cruz Loma, Pichincha, Ecuador
}

\author{
Diego Céspedes y Violeta Rafael \\ Laboratorio de Genética Evolutiva, Escuela de Ciencias Biológicas, Pontificia Universidad Católica \\ del Ecuador, Quito, Ecuador \\ diego_cesp@hotmail.com; vrafael@puce.edu.ec
}

Recibido: 28, 06, 2013; aceptado: 04, 10, 2013

\begin{abstract}
RESUMEN.- La quebrada de Cruz Loma, situada en el lado oriental del volcán Pichincha, provincia de Pichincha, fue muestreada durante el año 2008 y comienzos del 2009 con la finalidad de poder determinar la composición de las especies del género Drosophila. Se fijaron 13 puntos de colecta, y se utilizaron trampas de banana-levadura. Los muestreos se realizaron desde los 3100 hasta $4000 \mathrm{~m}$ de altitud. Los ecosistemas muestreados fueron el páramo propiamente dicho (3 500-4 $100 \mathrm{~m}$ ) cubierto por los géneros Calamagrostis y Festuca y el subpáramo (3 000-3 500 m) compuesto en su mayor parte por vegetación arbustiva. Un total de 713 individuos de diferentes especies del género Drosophila fueron capturados. No se recolectaron especímenes sobre los $3850 \mathrm{~m}$. La mayor cantidad de individuos y especies se registraron a 3100 m (12 especies). Los meses con mayor número de capturas fueron julio (239 especímenes) y octubre (236 especímenes). Así mismo, en octubre se recolectaron 14 especies. Las especies más abundantes fueron Drosophila rucux, con 193 individuos capturados a lo largo del año, desde los 3100 hasta los 3775 m, y Drosophila ecuatoriana con 129 individuos capturados desde los 3100 hasta los $3550 \mathrm{~m}$ de altitud. Además y por primera vez en Ecuador se colectaron especímenes de Drosophila hyalipennis Duda (1927) a 3 325-3 $400 \mathrm{~m}$.
\end{abstract}

PALABRAS CLAVES: Cruz Loma, diversidad, Drosophila, nuevo registro

\begin{abstract}
The Cruz Loma gorge on the eastern slope of the Pichincha volcano in the province of Pichincha, was sampled during 2008 and early 2009, in order to determine the composition of the genus Drosophila. Thirteen collection points were determined. Traps with banana and yeast were used. Samples were taken from 3100 to $4000 \mathrm{~m}$ altitude. Ecosystems sampled were the páramo (3 500-4 $100 \mathrm{~m}$ ) covered by the genera Calamagrostis and Festuca and subpáramo (3 000-3 $500 \mathrm{~m}$ ) composed mainly of shrub forest. A total of 713 individuals of different species of Drosophila were captured.
\end{abstract}


No specimens were collected over $3850 \mathrm{~m}$. Most individuals and species were recorded at $3100 \mathrm{~m}$ (12 species). The months with the larger amounts of captured flies were July (239 specimens) and October (236 specimens). October was the month where the greatest number of species (14) were captured. Most abundant species were Drosophila rucux, (193 individuals captured throughout the year, from 3100 up to $3775 \mathrm{~m}$ ), and Drosophila ecuatoriana (129 individuals captured from 3100 up to 3550 m altitude). Also, Drosophila hyalipennis Duda (1927) was also found for the first time in Ecuador at $3325-3400 \mathrm{~m}$.

KEYWORDS: Cruz Loma, diversity, Drosophila, new record

\section{INTRODUCCIÓN}

La familia Drosophilidae consta de 73 géneros y más de 3950 especies actualmente descritas, de las cuales la mayoría se encuentran en la Región Tropical (Brake y Bachli, 2008). El género Drosophila es el más abundante dentro de la familia y comprende alrededor del $53 \%$ del total de especies; de ellas algunas son endémicas otras subcosmopolitas y varias poseen una distribución cosmopolita (Mateus et al., 2006).

Las especies del género Drosophila tienen una distribución altitudinal muy amplia, ya que, se puede encontrar desde el nivel del mar hasta más allá de los 3000 msnm (Dillon \& Frazier, 2006). Dobzhansky (1956) utilizó en sus investigaciones a $D$. pseudoobscura recolectada a $3200 \mathrm{msnm}$ junto con $D$. persimilis y $D$. occidentalis. Además, D. ananassae se ha logrado capturar a una altitud de 5123 msnm en las laderas de Los Himalayas (Khare et al., 2002). En el Ecuador, estudios realizados en "El Refugio de Vida Silvestre Pasochoa" a $3200 \mathrm{msnm}$ han revelado la existencia de numerosas especies nuevas del género
Drosophila (Vela \& Rafael, 2003). Así mismo, en una colecta realizada en el páramo de "La Virgen" a 4100 msnm (bosque de Polylepis) se han capturado drosófilas y entre ellas $D$. malerkotliana considerada como una especie invasiva (Rafael, 2007); además de varias especies endémicas como las reportadas por Figuero et al, 2012.

Las especies para poder mantener poblaciones viables en las altas montañas, deben localizar comida, ser una pareja reproductiva y evadir a los depredadores, mientras enfrentan condiciones como: baja temperatura, baja concentración de oxígeno y otros factores que reducen la efectividad en la locomoción de estos insectos ectotérmicos (Dillon y Frazier, 2006).

El objetivo del presente estudio, fue analizar la variación de la diversidad del género Drosophila a lo largo de un gradiente altitudinal (3 100 a $4000 \mathrm{msnm}$ ) de la quebrada de Cruz Loma (sector norte del Teleférico de Quito) del Bosque Protector Pichincha. 


\section{MATERIALES Y MÉTODOS}

El área de estudio está ubicada en el Bosque Protector Pichincha situado en el flanco oriental de la cordillera occidental de Los Andes y corresponde a la quebrada y loma denominada Cruz Loma (78³1'17.2”'W 0¹1’22"S). El área de colecta comprendió un rango altitudinal entre los 3100 y los $4000 \mathrm{msnm}$.

En esta quebrada se encuentran varios tipos de vegetación: 1) El páramo propiamente dicho se encuentra ubicado aproximadamente desde los 3500 hasta los 4100 msnm y posee una vegetación de pajonal dominada principalmente por los géneros Calamagrostis y Festuca (Luteyn, 1999); 2) el subpáramo es una zona de transición situada entre los 3000 y 3500 msnm compuesta en su mayor parte por vegetación arbustiva (Luteyn, 1999); 3) el bosque altoandino comprendido entre los 3400 y 4300 msnm se encuentra compuesto por árboles de 3 a 10 metros de alto e interrumpido por grandes extensiones de pajonales.

La recolección de individuos se realizó durante el año 2008 e inicios del 2009. Para el muestreo se utilizaron trampas hechas con una botella plástica de $500 \mathrm{ml}$ con agujeros en la parte superior y con una cubierta plástica para protegerla de la lluvia. Como cebo se utilizó plátano fermentado con una solución de levadura al $10 \%$. Se colocaron un total de 78 trampas a lo largo de la ladera y la colecta se realizó luego de 14 y 21 días de haber colocado las trampas en el campo. Los individuos vivos fueron capturados con un aspirador entomológico y colocados en tubos con medio de cultivo; mientras que, los muertos fueron retirados de las trampas y guardados en tubos con etanol al $70 \%$.

Los individuos capturados vivos fueron separados por sexo. Los machos fueron utilizados para la identificación taxonómica, y con las hembras se fundaron isolíneas para obtener descendencia. La identificación taxonómica se realizó mediante el análisis de la genitalia interna y externa. Para obtener las estructuras de la genitalia tanto del macho como de la hembra, se separaron los últimos segmentos abdominales y se hirvieron durante 10 minutos en una solución de $\mathrm{KOH}$ al 10 \%; luego se realizó la disección; el falo, epandrio e hipandrio del macho fueron colocados en glicerol y observados al microscopio. En las hembras se separó el ovipositor y las espermatecas que fueron colocados en glicerol al $60 \%$.

\section{RESULTADOS}

Se colectaron un total de 713 individuos del género Drosophila que pertenecen a 16 especies distribuidas en siete grupos de especie.

Aunque el muestreo se realizó hasta los 4000 msnm, no se logró colectar individuos a partir de los $3850 \mathrm{msnm}$ en ninguno de los meses en los cuales se llevó a cabo las colectas.

En relación con el número de especies en cada una de las estaciones, se observa 
que en la estación ubicada a los 3100 (12 especies) la cual se reduce hasta llegar msnm se encuentra la mayor diversidad a cero especies a los $3850 \mathrm{msnm}$ (Tabla 1).

\section{Tabla 1}

Número total de individuos del género Drosophila capturadas en las diferentes altitudes de la Quebrada de Cruz Loma, Provincia de Pichincha, Ecuador

\begin{tabular}{|c|c|c|c|c|c|c|c|c|c|c|c|c|c|c|}
\hline Especies & 할 & $\frac{10}{m}$ & గ్రి & क्षి & 윰 & $\begin{array}{l}\text { N } \\
\text { 今े }\end{array}$ & 占 & $\begin{array}{l}\text { त్రి } \\
\text { है }\end{array}$ & 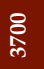 & $\underset{\mathrm{N}}{\mathrm{N}}$ & $\begin{array}{l}\text { ஜ̂ } \\
\text { के } \\
\text { in }\end{array}$ & สू & ఫे & TOTAL \\
\hline \multicolumn{15}{|l|}{ Grupo asiri } \\
\hline D. asiri Vela \& Rafael, 2005 & - & - & - & 1 & - & - & - & - & - & - & - & - & - & 1 \\
\hline D. yuragyacum Figuero et al., 2012 & - & - & - & - & - & - & 7 & - & - & - & - & - & - & 7 \\
\hline \multicolumn{15}{|l|}{ Grupo busckii } \\
\hline D. busckii Coquillett, 2001 & 1 & - & - & - & - & - & - & - & - & - & - & - & - & 1 \\
\hline \multicolumn{15}{|l|}{ Grupo flavopilosa } \\
\hline D. korefae Vela \& Rafael, 2004 & 2 & 5 & - & 1 & - & - & - & - & - & - & - & - & - & 8 \\
\hline D. ogradi Vela \& Rafael, 2004 & 29 & 1 & - & - & - & - & - & - & - & - & - & - & - & 30 \\
\hline \multicolumn{15}{|l|}{ Grupo guarani } \\
\hline D. ecuatoriana Vela \& Rafael, 2004 & 57 & 41 & 18 & 3 & 4 & 4 & 2 & - & - & - & - & - & - & 129 \\
\hline \multicolumn{15}{|l|}{ Grupo mesophragmatica } \\
\hline D. amaguana Vela \& Rafael, 2004 & 69 & 5 & 4 & - & - & 3 & 1 & - & - & - & - & - & - & 82 \\
\hline D. mesophragmatica Duda, 1927 & 1 & 17 & 2 & 50 & 19 & 11 & - & - & 1 & - & - & - & - & 101 \\
\hline D. rucux Céspedes \& Rafael, 2012 & 1 & 1 & 9 & 96 & 31 & 14 & 2 & 27 & 10 & 2 & - & - & - & 193 \\
\hline D. yanayuyu Céspedes \& Rafael, 2012 & 2 & - & - & - & - & 4 & - & - & - & - & - & - & - & 6 \\
\hline \multicolumn{15}{|l|}{ Grupo onychophora } \\
\hline D. hyalipennis Duda, 1927 & - & - & - & 2 & 1 & - & - & - & - & - & - & - & - & 3 \\
\hline \multicolumn{15}{|l|}{ Grupo tipunctata } \\
\hline D. ninarumi Vela \& Rafael, 2005 & 23 & 2 & 1 & - & - & - & - & - & - & - & - & - & - & 26 \\
\hline D. pasochoensis Vela \& Rafael, 2001 & 18 & 4 & 4 & - & 2 & - & - & - & - & - & - & - & - & 28 \\
\hline D. condorhuachana Céspedes \& Rafael, 2012 & 73 & 4 & 13 & 1 & 1 & - & - & - & - & - & - & - & - & 92 \\
\hline \multicolumn{15}{|l|}{ Especies no agrupadas } \\
\hline D. apag Vela \& Rafael, 2005 & 3 & - & - & - & - & - & - & - & - & - & - & - & - & 3 \\
\hline D. condormachay Vela \& Rafael, 2005 & - & - & - & 2 & 1 & - & - & - & - & - & - & - & - & 3 \\
\hline TOTAL DE INDIVIDUOS & 279 & 80 & 51 & 156 & 59 & 36 & 12 & 27 & 11 & 2 & - & - & - & 713 \\
\hline
\end{tabular}

De los cuatro meses en los cuales se realizaron las colectas, julio y octubre fueron los meses de mayor captura, 239 y 236 individuos respectivamente; así mismo, octubre fue el mes en el cual más especies se registraron (14 especies). Además, en enero se recolectó un menor número de individuos; a pesar de ello, el número de especies presentes es similar al de los otros meses (Tabla 2).
Las especies mejor representadas por número de individuos y que están presentes en gran parte de el gradiente altitudinal son: Drosophila rucux, con 193 individuos a lo largo del año (Tabla 1), distribuidos desde los 3100 hasta los 3775 msnm y D. ecuatoriana con 129 individuos colectados desde los 3100 hasta los $3550 \mathrm{msnm}$. 
Tabla 2

Especies del género Drosophila capturadas en la Quebrada de Cruz Loma en cada mes de colecta

\begin{tabular}{|c|c|c|c|c|}
\hline \multicolumn{2}{|l|}{ Especies } & \multicolumn{2}{|c|}{ Meses } & \multirow[b]{2}{*}{ Enero } \\
\hline Grupo asiri & Abril & Julio & Octubre & \\
\hline D. asiri & - & - & 1 & - \\
\hline D. yuragyacum & - & 6 & 1 & - \\
\hline \multicolumn{5}{|l|}{ Grupo busckii } \\
\hline D. busckii & 1 & - & - & - \\
\hline \multicolumn{5}{|l|}{ Grupo flavopilosa } \\
\hline D. korefae & - & - & 8 & - \\
\hline D. ogradi & - & 14 & 15 & 1 \\
\hline \multicolumn{5}{|l|}{ Grupo guarani } \\
\hline D. ecuatoriana & 68 & 26 & 28 & 7 \\
\hline \multicolumn{5}{|c|}{ Grupo mesophragmatica } \\
\hline D. amaguana & 3 & 34 & 39 & 6 \\
\hline D. mesophragmatica & 21 & 34 & 44 & 2 \\
\hline D. rucux & 75 & 50 & 58 & 10 \\
\hline D. yапауиуи & 2 & - & 1 & - \\
\hline \multicolumn{5}{|l|}{ Grupo onychophora } \\
\hline D. hyalipennis & 3 & - & - & - \\
\hline \multicolumn{5}{|l|}{ Grupo tipunctata } \\
\hline D. ninarumi & - & 3 & 17 & 6 \\
\hline D. pasochoensis & 4 & 15 & 8 & 1 \\
\hline D. condorhuachana & 20 & 57 & 9 & 6 \\
\hline \multicolumn{5}{|c|}{ Especies no agrupadas } \\
\hline D. apag & - & - & 3 & - \\
\hline D. condormachay & - & - & 4 & 2 \\
\hline No. de especies & 9 & 9 & 14 & 9 \\
\hline Total de individuos & 197 & 239 & 236 & 41 \\
\hline
\end{tabular}


De un total de 16 especies del género Drosophila capturadas en la quebrada de Cruz Loma, D. hyalipennis es el único nuevo registro para el Ecuador.

\section{DISCUSIÓN}

En la quebrada de Cruz Loma se capturaron 713 individuos pertenecientes a 16 especies del género Drosophila de los subgéneros, Dorsilopha y Drosophila.

Dentro del subgénero Dorsilopha la única especie que se encontró fue Drosophila busckii; esta especie es considerada según Patterson y Stone (1952) como una de las ocho especies de Drosophila que son cosmopolitas junto con D. ananassae, D. hydei, D. immigrans, D. malerkotliana, D. melanogaster, D. repleta y D. simulans. Las especies cosmopolitas se encuentran presentes comúnmente en lugares que han tenido influencias antrópicas; sin embargo, especies como D. simulans, $D$. inmigrans y $D$. ananassae se las puede encontrar en lugares sin ningún disturbio pero en densidades muy bajas (Ferreira \& Tidon, 2005). En este caso se encontró un solo individuo de D. busckii en el punto de colecta más bajo del estudio (3 $100 \mathrm{msnm}$ ); este lugar es el más cercano a la estación base del Teleférico de Quito, por lo cual, se podría pensar que existe alguna influencia antrópica. Por lo tanto, el hecho de haber encontrado a Drosophila busckii en el área de estudio, no significa necesariamente que se trate de un lugar intervenido pues la densidad de esta especie es sumamente baja; además es posible considerar una contaminación del tubo de colecta en el laboratorio en el momento del análisis.
Como se esperaba, la mayor cantidad de especies y de individuos se encuentran presentes en el punto de muestreo más bajo del estudio (3 $100 \mathrm{msnm}$ ). El número desciende rápidamente hasta llegar a cero a los 3850 m en donde el área de muestreo se caracteriza principalmente por la presencia de pajonal y un número muy reducido de arbustos; estas condiciones dificultarían el establecimiento de poblaciones debido a la ausencia de un refugio contra el viento y la lluvia que es necesario para combatir el frío, obtener comida y tener lugares de ovoposición y cópula.

Aunque se realizaron varios análisis estadísticos (ANOSIM, SIMPER, GLM, entre otros), no fue posible encontrar una relación directa entre la distribución de especies a lo largo de la quebrada y las condiciones bióticas y abióticas presentes en la misma. Probablemente debido al gran número de ausencias registradas en los puntos de mayor altitud. Sin embargo, mediante observación directa, se puede afirmar que ciertas especies muestran preferencia por distintos factores; por ejemplo: D. amaguana es una especie de gran tamaño que se la encuentra principalmente en lugares con vegetación alta, con poca luminosidad y alta humedad; D. mesophragmatica ha sido capturada en lugares con vegetación de tipo arbustiva y con luz solar fuerte. Especies como D. ecuatoriana cuya abundancia decrece cuando la altitud se incrementa, sugiere que la temperatura es el factor limitante de su distribución. Para otras especies es difícil determinar las condiciones favorables para 
su establecimiento debido a su distribución muy restringida como $D$. asiri y $D$. apag o por el contrario especies con distribuciones amplias como D. rucux.

\section{REFERENCIAS BIBLIOGRÁFICAS}

Brake I y Bächli G. 2008. World Catalogue of Insects, Volumen 9, Apollo Books, Stenstrup, Denmark.

Dillon M y Frazier M. 2006. Drosophila melanogaster locomotion in cold thin air. The Journal of Experimental Biology, 209: 364-371.

Dobzhansky T. 1956. Genetics of natural populations. XXV. Genetic changes in populations of Drosophila pseudoobscura and D. persimilis in some locations in California. Evolution, 10: 82-92.

Ferreira L y Tidon R. 2005. Colonizing potential of Drosophilidae (Insecta, Diptera) in environments with different grades of urbanization. Biodiversity and Conservation, 14: 1809-1821.

Figuero ML, Rafael V y Céspedes D. 2012. Grupo D. asiri (Diptera, Drosophilidae), un nuevo grupo de especies andinas con la descripción de dos nuevas especies y la redescripción de Drosophila asiri. Iheringia, Série Zoologia, 102 (1): 33-42.
Khare PV, Burnabas RJ, Kanojiya M, Kulkarni AD y Yoshi DS. 2002. Temperature dependent eclosion rhythmicity in the high altitude himalayan strains of Drosophila ananassae. Chronobiology International, 19: 10411052.

Luteyn JL. 1999. Páramos: a Checklist of Plant Diversity, Geographical Distribution, and Botanical Literature. The New York Botanical Garden Press, 84, Bronx, New York.

Mateus RP, Buschini MLT y Sene FM. 2006. The Drosophila community in xerophytic vegetations of the upper Parana-Paraguay River Basin. Brazilian Journal of Biology, 66 (2B): 719-729.

Patterson JT y Stone WS. 1952. Evolution in the genus Drosophila. Macmillan, New York. 610 pp.

Rafael V. 2007. Drosophila malerkotliana y Zaprionus indianus (Diptera, Drosophilidae) invaden poblaciones ecuatorianas de Drosophila. Revista Ecuatoriana de Medicina y Ciencias Biológicas, 38 (1, 2): 30-43.

Vela D y Rafael V. 2003. Estudios sobre la diversidad del género Drosophila (Diptera, Drosophilidae) en el Bosque Pasochoa de la provincia de Pichincha-Ecuador. Revista de la Pontificia Universidad Católica del Ecuador, 71: 117-127. 